\title{
A backscattering formula for acoustic transducers
}

\author{
Abdullah Atalar \\ Edward L. Ginzton Laboratory, W. W. Hansen Laboratories of Physics, Stanford University, Stanford, \\ California 94305
}

(Received 30 November 1979; accepted for publication 26 February 1980)

\begin{abstract}
A backscattering formula for a single transducer geometry is derived. It expresses the output voltage of the transducer in terms of the angular spectra of scalar and vector acoustic potentials on a plane. The formulation is suitable for acoustic problems involving wide angular spectrum beams. The derived formula gives a simple expression for the problem of a circular transducer facing a plane reflector. The output voltage of a transducer receiving the backscattered waves from an arbitrary size spherical flaw is also presented.
\end{abstract}

PACS numbers: 43.20.Fn, 03.40.Dz, 43.20.Bi, 61.20.Lc

\section{INTRODUCTION}

$\mathrm{Kino}^{1}$ and $\mathrm{Auld}^{2}$ have applied the generalized reciprocity relations to the calculation of acoustic wave scattering coefficients. They derived a scattering formula that relates the scattering coefficient at the electrical terminals of an acoustic transducer to a surface integral evaluated at a surface around the flaw. Using this integral along with Born or quasistatic approximations it is possible to arrive at closed form expressions for some simple geometries. This scattering formula is valid for both bulk and surface wave transducers.

In this paper we consider a single transducer geometry. We start from the "Kino-Auld general scattering formula" and derive an expression that is written in terms of angular spectra of scalar and vector potentials instead of the particle velocity and stress field. This form of the expression is simpler more easily adopted to many backscattering problems. It is especially convenient to use in numerical calculations. The formulation makes it possible to obtain results for highly convergent, i.e., wide spectrum, acoustic beams.

Two examples are treated to illustrate the use of the derived expression. The first is the calculation of the output of a circular transducer as a function of the distance between the transducer and a parallel planar reflector. The effect of tilting the planar reflector with respect to the transducer is also considered. The second example is concerned with the scattering from a spherical flaw. A simple formula is derived that expresses the output voltage in terms of the potential

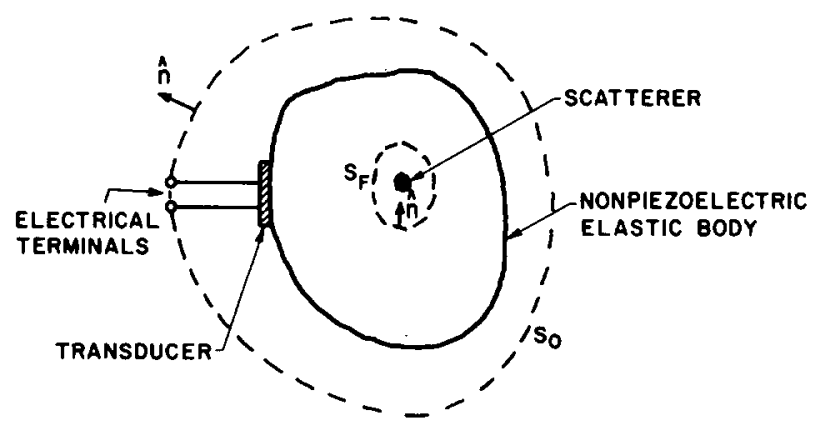

FIG. 1. Scattering geometry of Kino-Auld formula suitable for a single transducer. field of the transducer and a function that is dependent on the size and character of the spherical flaw.

\section{DERIVATION OF THE FORMULA}

We consider a transducer bonded to an elastic body used as both transmitter and receiver as shown in Fig. 1. For this single transducer geometry the Kino-Auld formula can be stated with the notation of Auld $^{2}$ as follows:

$$
\delta F=\frac{1}{4 P} \int_{S_{i}}\left(\mathbf{v}_{1} \cdot \mathrm{T}_{2}-\mathbf{v}_{2} \cdot \mathrm{T}_{1}\right) \cdot \hat{n} d S,
$$

where $\mathrm{v}$ is the particle velocity vector, $\mathrm{T}$ is the stress tensor, $\hat{n}$ is the inward-directed normal to the surface $S_{F}$ surrounding the scatterer. The field quantities under the integral with subscript 1 are excited in the absence of the scatterer and those with subscript 2 are excited in the presence of the scatterer. $P$ is the incident power at the transducer terminals. $\delta F$ is the change in the electrical reflection coefficient due to the presence of the scatterer. The left-hand side of this equation is the contribution of the outer integration surface $\left(S_{0}\right)$ of

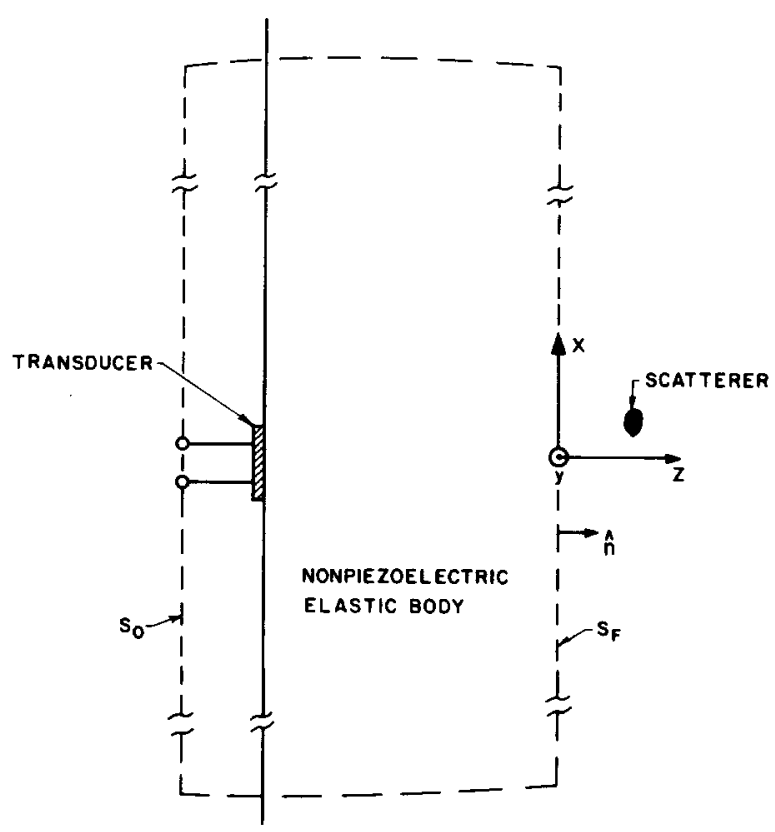

FIG. 2. Scattering geometry and coordinate system used in formulation Note that the flaw is not included within the integration surface. 
Fig. 1. The inner integration surface $S_{F}$ gives rise to the right-hand side of the equation.

We change the geometry to that shown in Fig. 2. The nonpiezoelectric elastic body is assumed to cover the half space of the body. A single connected integration surface is selected with a face coincident at $Z=0$ plane. The surface extends to a large enough diameter so that the acoustic fields are negligible at that point. Therefore the $Z=0$ plane can be treated as the integration surface $S_{F}$ of Eq. (1). The left-hand side of the equation is produced by the integration over the surface $S_{0}$, which intersects the electrical terminals of the transducer. We select our two field solutions as follows. Subscript 1 refers to the solution with no reflector or flaw present in $Z \geqslant 0$ half space, and hence all the field components propagate in the $+z$ direction. Subscript 2 represents the solution with a reflector or flaw present at some distance from the $Z=0$ plane. In this case there are waves propagating in both $+z$ and $-z$ directions. In the formulation we assume that the reflection coefficient at the electrical terminal is zero when the reflector is not present. In practice this can be realized by the pulse-echo technique and time gating. Therefore, the change in the reflection coefficient $\delta F$ is really equal to the normalized amplitude of the echo pulse from the flaw: $V$.

We represent the waves that are propagating in the $+z$ direction with a " + " superscript and those propagating in the $-z$ direction (backscattered) with a " - " superscript. We neglect the multiple reflections between the reflector object and the transducer, or alternatively we use the pulseecho technique and look at the first reflection only. Thus we write

$\mathbf{v}_{1}=\mathbf{v}^{+}, \quad \mathbf{v}_{2}=\mathbf{v}^{+}+\mathbf{v}^{-}, \quad \mathbf{T}_{1}=\mathbf{T}^{+}, \quad \mathbf{T}_{2}=\mathbf{T}^{+}+\mathbf{T}^{-}$.

Substituting these in Eq. (1), one finds

$$
V=\frac{1}{4 P} \int_{S_{i}}\left(\mathbf{v}^{-} \cdot \mathbf{T}^{+}-\mathbf{v}^{+} \cdot \mathbf{T}^{-}\right) \cdot \hat{n} d S .
$$

Since we are integrating over the surface at $z=0$, we have $\hat{n}=\hat{a}_{z}$ and $d S=d x d y$. Therefore we can write Eq. (2) more explicitly as

$$
\begin{aligned}
V= & \frac{1}{4 P} \int_{-\infty}^{\infty} \int\left(v_{x}^{-} T_{z x}^{+}+v_{y}^{-} T_{z y}^{+}+v_{z}^{-} T_{z z}^{+}\right. \\
& \left.-v_{x}^{+} T_{z x}^{-}-v_{y}^{+} T_{z y}^{-}-v_{z}^{+} T_{z z}^{-}\right) d x d y .
\end{aligned}
$$

At this point we assume that the propagation medium for $Z \leqslant 0$ is homogeneous and isotropic. We can now write the stress components in terms of the velocity components and elastic parameters of the medium. ${ }^{3}$ With $\exp (-j \omega t)$ time dependence we find

$$
\begin{aligned}
V= & \frac{j}{4 \omega P} \int_{-\infty}^{\infty} \int\left[v_{x}^{-} c_{44}\left(v_{x, z}^{+}+v_{z, x}^{+}\right)\right. \\
& +v_{y}^{-} c_{44}\left(v_{z, y}^{+}+v_{y, z}^{+}\right)+v_{z}^{-} c_{11} v_{z, z}^{+}+v_{z}^{-} c_{12}\left(v_{x, x}^{+}+v_{y, y}^{+}\right)
\end{aligned}
$$

$$
\begin{aligned}
& -v_{x}^{+} c_{44}\left(v_{x, z}^{-}+v_{z, x}^{-}\right)+v_{y}^{+} c_{44}\left(v_{z, y}^{-}+v_{y, z}^{-}\right) \\
& \left.-v_{z}^{+} c_{11} v_{z, z}^{-}-v_{z}^{+} c_{12}\left(v_{x, x}^{-}+v_{y, y}^{-}\right)\right] d x d y,
\end{aligned}
$$

where we used the usual suffix notation $\partial v_{i} / \partial x_{j}=v_{i j}$ and the elastic constants $c_{11}, c_{44}, c_{12}\left(=c_{11}-2 c_{44}\right)$ of the medium.

It is convenient to decompose the vector field $\mathbf{v}$ into a scalar potential $\phi$ and a vector potential $\psi$ associated with longitudinal and shear waves, respectively. ${ }^{4}$

$\mathbf{v}=\nabla \phi+\nabla \times \psi$, where $\phi$ and $\psi$ satisfy the Helmholtz equation $\nabla^{2} \phi+k_{0}^{2} \phi=0$ and $\nabla^{2} \psi+k_{0}^{\prime 2} \psi=0$. Thus the individual components of the particle velocity are written as

$$
\begin{aligned}
& v_{x}=\phi_{, x}+\psi_{z, y}-\psi_{y, z}, \\
& v_{y}=\phi_{, y}+\psi_{x, z}-\psi_{z, x}, \\
& v_{z}=\phi_{, z}+\psi_{y, x}-\psi_{x, y},
\end{aligned}
$$

where we used an additional suffix notation $\partial \phi / \partial x_{i}=\phi_{, i}$. To reduce the expressions into a simple form we will switch to angular spectrum domain. The angular spectrum approach is shown to be a powerful technique for wave problems. It is used successfully in optical problems ${ }^{5,6}$ as well as some acoustic problems. ${ }^{7.8}$

Let $\Phi$ and $\Psi$ show the angular spectra of $\phi$ and $\psi$, respectively. To establish the notation we write the relationship between the transform pair $\phi \leftrightarrow \Phi$ as

$$
\begin{aligned}
\phi(x, y)= & {\left[1 /(2 \pi)^{2}\right] \int_{-\infty}^{\infty} \int \Phi\left(k_{x}, k_{y}\right) } \\
& \times \exp \left[j\left(k_{x} x+k_{y} y\right)\right] d k_{x} d k_{y}
\end{aligned}
$$

and

$\Phi\left(k_{x}, k_{y}\right)=\int_{-\infty}^{\infty} \int \phi(x, y) \exp \left[-j\left(k_{x} x+k_{y} y\right)\right] d x d y$.

Using the properties of Fourier transformation ${ }^{5}$ and

Eq. (5) one can find the following transform pairs:

$$
\begin{aligned}
& v_{x}^{\mp} \leftrightarrow j k_{x} \Phi \mp+j k_{y} \Psi_{z}^{\mp} \pm j k_{z}^{\prime} \Psi_{y}^{\mp}, \\
& v_{y}^{\mp} \leftrightarrow j k_{y} \Phi \mp \mp j k_{z}^{\prime} \Psi_{x}^{\mp}-j k_{x} \Psi_{z}^{\mp}, \\
& v_{z}^{\mp} \leftrightarrow \mp j k_{z} \Phi \mp+j k_{x} \Psi_{y}^{\mp}-j k_{y} \Psi_{x}^{\mp}, \\
& v_{x, z}^{\mp} \leftrightarrow \pm k_{x} k_{z} \Phi \mp \pm k_{y} k_{z}^{\prime} \Psi_{z}^{\mp}+k_{z}^{\prime 2} \Psi_{y}^{\mp}, \\
& v_{z, z}^{\mp} \leftrightarrow-k_{z}^{2} \Phi \mp \pm k_{x} k_{z}^{\prime} \Psi_{y}^{\mp} \mp k_{y} k_{z}^{\prime} \Psi_{x}^{\mp}, \\
& v_{x, x}^{\mp} \leftrightarrow-k_{x}^{2} \Phi \mp-k_{x} k_{y} \Psi_{z}^{\mp} \mp k_{x} k_{z}^{\prime} \Psi_{y}^{\mp},
\end{aligned}
$$

where $k_{z}=\left(k_{0}^{2}-k_{x}^{2}-k_{y}^{2}\right)^{1 / 2}$ and $k_{z}^{\prime}=\left(k_{0}^{\prime 2}\right.$ $\left.-k_{x}^{2}-k_{y}^{2}\right)^{1 / 2}$.

We now employ the Generalized Parseval's Theorem (see Appendix) on each term of Eq. (4) using the pairs found above. This operation converts the right-hand side of Eq. (4) to angular spectrum domain. After some tedious and lengthy algebra we arrive at

$$
\begin{aligned}
V= & \frac{-1}{8 \pi^{2} \omega P}\left(c_{11} k_{0}^{2} \int_{-\infty}^{\infty} \int k_{z} \Phi^{-}\left(k_{x}, k_{y}\right) \Phi^{+}\left(-k_{x},-k_{y}\right) d k_{x} d k_{y}+c_{44} \int_{-\infty}^{\infty} \int k_{z}^{\prime}\left[\Psi_{x}^{-}\left(k_{x}, k_{y}\right) \Psi_{y}^{-}\left(k_{x}, k_{y}\right) \Psi_{z}^{-}\left(k_{x}, k_{y}\right)\right]\right. \\
& \left.\times\left[\begin{array}{ccc}
k_{y}^{2}+k_{z}^{\prime 2} & -k_{x} k_{y} & k_{x} k_{z}^{\prime} \\
-k_{x} k_{y} & k_{x}^{2}+k_{z}^{\prime 2} & k_{y} k_{z}^{\prime} \\
k_{x} k_{z}^{\prime} & k_{y} k_{z}^{\prime} & k_{x}^{2}+k_{y}^{2}
\end{array}\right]\left[\begin{array}{l}
\Psi_{x}^{+}\left(-k_{x},-k_{y}\right) \\
\Psi_{y}^{+}\left(-k_{x},-k_{y}\right) \\
\Psi_{z}^{+}\left(-k_{x},-k_{y}\right)
\end{array}\right] d k_{x} d k_{y}\right)
\end{aligned}
$$


This is our final result. It expresses the normalized transducer output voltage in terms of the angular spectra of the potential functions. The first integral is the contribution of the longitudinal waves and the second is due to shear waves. For a liquid medium or for a pulse system where only the longitudinal wave echos are gated, only the first integral is to be considered. Note the absence of cross terms between the scalar potential $\Phi$, and the vector potential $\Psi$. This simply tells us that a transducer generating a purely shear (purely longitudinal) wave will not respond to incident longitudinal (shear) waves. In other words if a longitudinal wave transducer is responding to incident shear waves it must also be generating them when used as a transmitter.

Note that no assumption is made in the derivation for the position of the transducer with respect to the $Z=0$ plane. Hence this plane can be selected at any convenient location.

For the paraxial case (narrow angular spectrum condition) the first integral of Eq. (6) can be written approximately as

$V \cong \frac{-c_{11} k_{0}^{3}}{8 \pi^{2} \omega P} \int_{-\infty}^{\infty} \int \Phi^{-}\left(k_{x}, k_{y}\right) \Phi^{+}\left(-k_{x},-k_{y}\right) d k_{x} d k_{y}$,

where we used the approximation $k_{2} \simeq k_{0}$. Application of the Parseval theorem results in

$$
V=-\frac{c_{11} k_{0}^{3}}{2 \omega P} \int_{-\infty}^{\infty} \int \phi^{-}(x, y) \phi^{+}(x, y) d x d y .
$$

This expression is widely used to calculate the output voltage of a longitudinal wave transducer. Our derivation shows this expression is only valid under the paraxial case.

\section{APPLICATIONS}

In this section we present some examples to illustrate the use of the formula derived in the previous section.

\section{A. Example 1}

Suppose that we place a perfect planar reflector at a distance $l$ away from a circular longitudinal wave transducer of radius $a$. We would like to find the output voltage of the transducer as a function of $l$. Referring to Fig. 3(a) we select the $Z=0$ plane just near the reflector, i.e., a distance $l$ away from the tranducer. We write the scalar potential at the plane of the transducer as

$A \operatorname{circ}\left[\left(x^{2}+y^{2}\right)^{1 / 2} / a\right] \quad$ where $\operatorname{circ}(r)=\left\{\begin{array}{ll}1 & r \leqslant 1 \\ 0 & r>0\end{array}\right.$.

Hence the angular spectrum at the same plane is given by a jinc function: $2 \pi a^{2} A J_{1}\left[a\left(k_{x}^{2}+k_{y}^{2}\right)^{1 / 2} /\right] a\left(k_{x}^{2}+k_{y}^{2}\right)^{1 / 2}$.

The spectrum at $Z=0$ is, then, written as

$$
\Phi^{+}\left(k_{x}, k_{y}\right)=4 a^{2} A \operatorname{jinc}\left[(a / \pi)\left(k_{x}^{2}+k_{y}^{2}\right)^{1 / 2}\right] \exp \left(j k_{z} l\right) \text {, }
$$

where jinc $x=J_{1}(\pi x) / 2 x$ and the exponential takes care of the propagation for the distance $l$. Since the reflector is perfect, we have $\Phi^{-}\left(k_{x}, k_{y}\right)=\Phi^{+}\left(k_{x}, k_{y}\right)$. Thus the output voltage is found from Eq. (6) as

$$
V=\frac{-2 c_{11} k_{0}^{2} a^{4}}{\pi^{2} \omega P} A^{2} \int_{-\infty}^{\infty} \int k_{2} \exp \left(j 2 k_{z} l\right)
$$

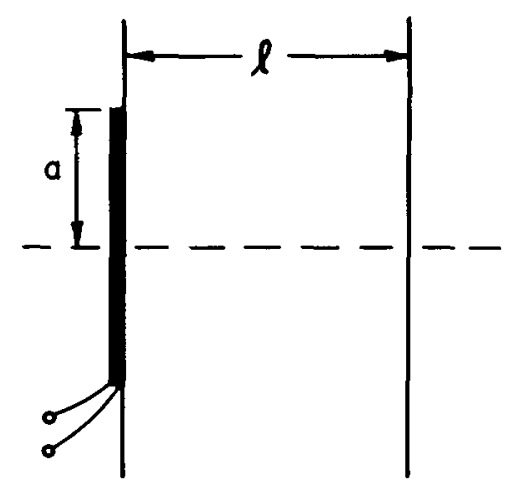

(a)

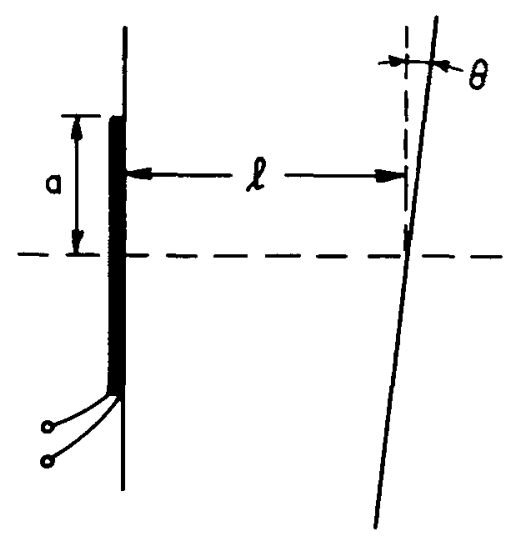

(b)

FIG. 3. Geometry for a circular transducer facing (a) parallel and (b) tilted reflector.

$$
\times \operatorname{jinc}^{2}\left(\frac{a}{\pi}\left(k_{x}^{2}+k_{y}^{2}\right)^{1 / 2}\right) d k_{x} d k_{y} .
$$

This integral can be simplified to read

$$
\begin{aligned}
V= & -\frac{4 c_{11} k_{0}^{2} a^{4}}{\pi \omega P} \\
& \times A^{2} \int_{0}^{k_{0}} k_{z}^{2} \operatorname{jinc}^{2}\left(\frac{a}{\pi}\left(k_{0}^{2}-k_{z}^{2}\right)^{1 / 2}\right) \exp \left(j 2 k_{z} l\right) d k_{z}
\end{aligned}
$$

or

$$
\begin{aligned}
V(l)= & -\frac{4 c_{11} k_{0}^{2} a^{4}}{\pi \omega P} A^{2} \\
& \times \mathscr{F}^{-1}\left\{k^{2} \operatorname{jinc}^{2}\left(\frac{a}{\pi}\left(k_{0}^{2}-k^{2}\right)^{1 / 2}\right)\right. \\
& \left.\times \operatorname{rect}\left(\frac{k-k_{0} / 2}{k_{0}}\right)\right\}\left.\right|_{x=2 l},
\end{aligned}
$$

where $\mathscr{F}^{-1}$ is the inverse Fourier transform operation and

$$
\operatorname{rect}(x)=\left\{\begin{array}{l}
1|x| \leqslant 1 / 2 \\
0|x|>1 / 2
\end{array}\right.
$$

Equation (8) shows that the output voltage of the transducer can be found by a single one-dimensional Fourier transformation.

A nonperfect (or a real) reflection process can be taken into account easily if we write $\Phi-\left(k_{x}, k_{y}\right)$ $=\mathscr{R}\left(k_{x} / k_{0}, k_{y} / k_{0}\right) \Phi^{+}\left(k_{x}, k_{y}\right)$. For an isotropic reflector 


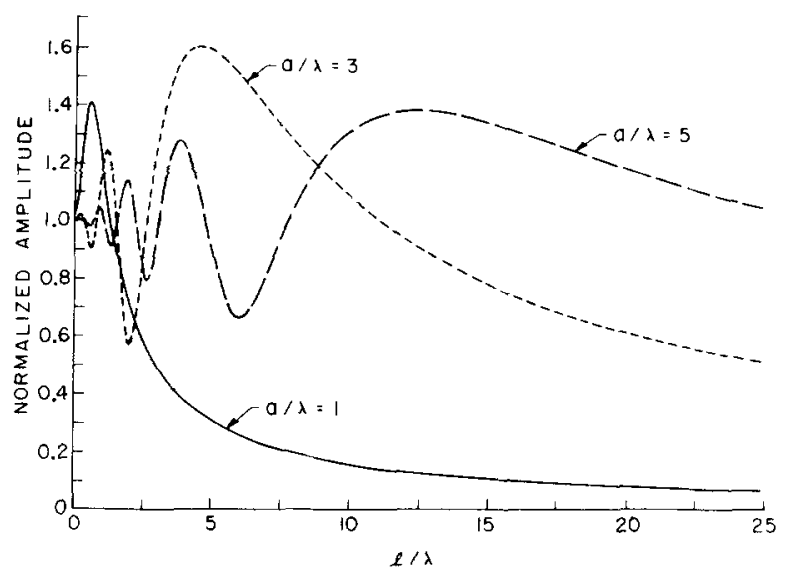

FIG. 4. Normalized output voltage of a circular transducer of radius $\lambda$ (solid), $3 \lambda$ (short dashed), $5 \lambda$ (long dashed), as a function of distance from a parallel reflector.

with a reflectance function $\mathscr{R}(\sin \theta)$ we have to add $\mathscr{R}\left[\left(k_{0}^{2}-k^{2}\right)^{1 / 2} / k_{0}\right]$ inside the brackets of Eq. (8).

Figure 4 shows the results of numerical calculations that utilizes Eq. (8) to find the transducer output voltage for various values of transducer radius.

Suppose that the reflector is tilted a small angle $\theta$, with respect to the $x$ axis as illlustrated in Fig. 3(b). Under the small angle approximation we write the reflected angular spectrum as

$$
\Phi^{-}\left(k_{x}, k_{y}\right)=\Phi^{+}\left(k_{x}-2 \theta k_{0}, k_{y}\right) \mathscr{R}\left(k_{x}-\theta k_{0}, k_{y}\right) .
$$

Therefore the normalized output voltage is given by

$$
\begin{aligned}
V= & -\frac{2 c_{11} k_{0}^{2} a^{4}}{\pi^{2} \omega P} A^{2} \\
& \times \int_{-\infty}^{\infty} \int k_{z} \operatorname{jinc}\left(\frac{a}{\pi}\left[\left(k_{x}-2 \theta k_{0}\right)^{2}+k_{y}^{2}\right]^{1 / 2}\right) \\
& \times \operatorname{jinc}\left(\frac{a}{\pi}\left(k_{x}^{2}+k_{y}^{2}\right)^{1 / 2}\right) \mathscr{R}\left(k_{x}-\theta k_{0}, k_{y}\right) \exp \left(j k_{z} l\right) \\
& \times \exp \left[j l\left(k_{0}^{2}-\left(k_{x}-2 k_{0} \theta\right)^{2}-k_{y}^{2}\right)^{1 / 2}\right] d k_{x} d k_{y}
\end{aligned}
$$

\section{B. Example 2}

As the second example we consider a spherical inhomogeneity placed at some distance to the transducer. This inhomogeneity could be in the form of a spherical cavity or an inclusion in the solid medium, or a solid reflector in a liquid medium.

Let us choose the $z=0$ plane parallel to the transducer and a distance $d$ away from the center of the sphere as depicted in Fig. 5(a). Let $\phi_{\text {ro }}(x, y)$ show the backscattered acoustic potential at this plane, when a plane wave of the form $\exp \left(j k_{0} z\right)$ [angular spectrum $\left.(2 \pi)^{2} \delta\left(k_{x}, k_{y}\right)\right]$ is incident on the spherical reflector.

Due to the spherical symmetry of the reflector, the backscattered field due to another plane wave of the form $\exp \left[j k_{0}(x \sin \theta+z \cos \theta)\right]$ can also be found. If $\theta$ is small we can write the backscattered acoustic potential at $z=0$ plane as

$$
\phi_{r \theta} \cong \phi_{\mathrm{ro}}(x+\theta d, y) \exp \left[-j k_{0} \theta x\right],
$$

and this approximation is demonstrated in Fig. 5(b). If $\Phi_{\mathrm{ro}}\left(k_{x}, k_{y}\right)$ is the angular spectrum of the field, then the angular spectrum of $\phi_{r \theta}$ is given approximately by $\Phi_{r o}\left(k_{x}+k_{0} \theta, k_{y}\right) \exp \left(j k_{x} \theta d\right)$.

We can generalize this further by writing the reflected angular spectrum for an incident plane wave with angular spectrum $(2 \pi)^{2} \delta\left(k_{x}-k_{y}^{\prime}, k_{y}-k_{y}^{\prime}\right)$ as $\Phi_{\text {ro }}\left(k_{x}+k_{x}^{\prime}, k_{y}+k_{y}^{\prime}\right) \exp \left[j\left(k_{x} d k_{x}^{\prime} / k_{0}, k_{y} d k_{y}^{\prime} / k_{0}\right)\right]$.

Now we can find the total reflected angular spectrum as the weighted sum of these individual angular spectra in the form of an integral:

$$
\begin{aligned}
\Phi^{-}\left(k_{x}, k_{y}\right)= & \int_{-\infty}^{\infty} \int \Phi^{+}\left(k_{x}^{\prime}, k_{y}^{\prime}\right) \Phi_{\mathrm{ro}}\left(k_{x}+k_{x}^{\prime}, k_{y}+k_{y}^{\prime}\right) \\
& \times \exp \left[j\left(d / k_{0}\right)\left(k_{x} k_{x}^{\prime}+k_{y} k_{y}^{\prime}\right)\right] d k_{x}^{\prime} d k_{y}^{\prime},
\end{aligned}
$$

where $\Phi^{+}\left(k_{x}^{\prime}, k_{y}^{\prime}\right)$ shows the weighting for individual incident plane waves. The last equation can be rearranged to read

$$
\begin{aligned}
& \Phi^{-}\left(k_{x}, k_{y}\right) \\
& =\exp \left[-j d\left(k_{x}^{2}+k_{y}^{2}\right) / 2 k_{0}\right] \int_{-\infty}^{\infty} \int \Phi^{+}\left(k_{x}^{\prime}, k_{y}^{\prime}\right) \\
& \times \exp \left[-j d\left(k_{x}^{\prime 2}+k_{y}^{\prime 2}\right) / 2 k_{0}\right] \Phi_{\mathrm{ro}}\left(k_{x}+k_{x}^{\prime}, k_{y}+k_{y}^{\prime}\right) \\
& \times \exp \left\{j d\left[\left(k_{x}+k_{x}^{\prime}\right)^{2}+\left(k_{y}+k_{y}^{\prime}\right)^{2}\right] / 2 k_{0}\right\} d k_{x}^{\prime} d k_{y}^{\prime} .
\end{aligned}
$$

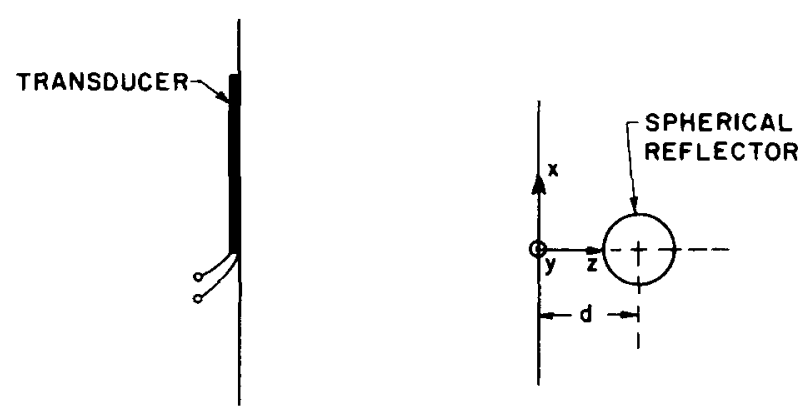

(a)

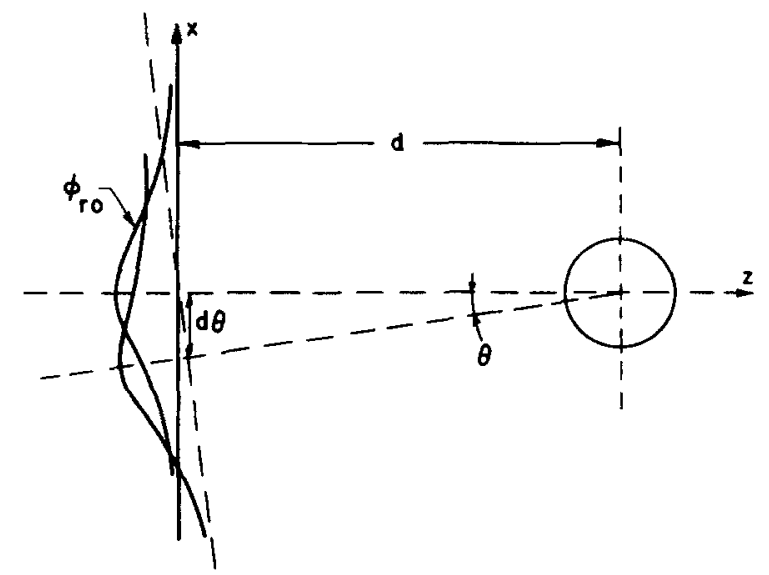

(b)

FIG. 5. (a) Geometry coordinate system used for the analysis of spherical flaw problem; (b) Illustration of backscattered waves when a spherical flaw is illuminated by plane waves. $\phi_{r}$ is the backscattered wave at $z=0$ when the incident plane wave is directed in $+z$ direction. 


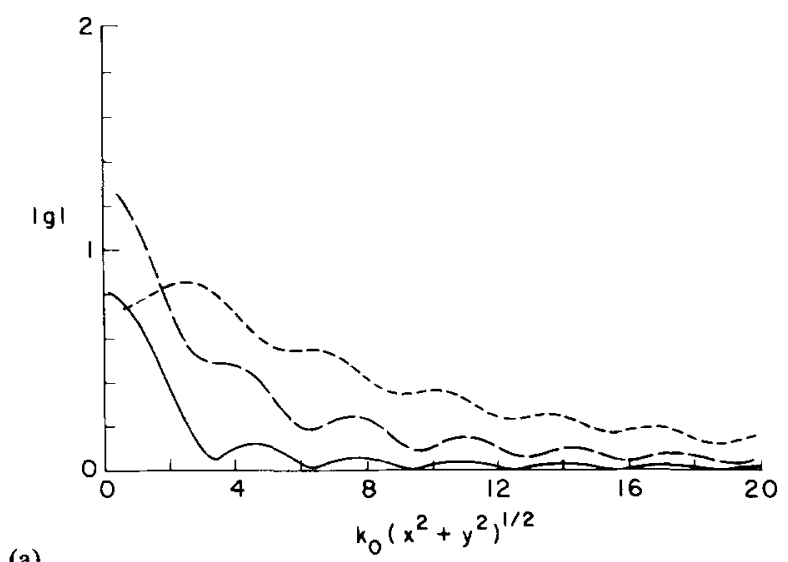

(a)

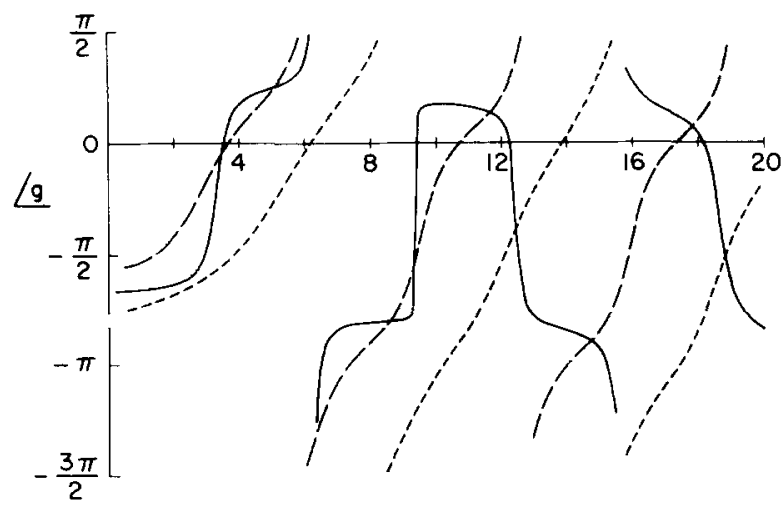

(b)

FIG. 6. (a) Amplitude and (b) phase of the function $g$ as a function of radial distance. Curves are for a YAG crystal containing a spherical cavity of radius $2 \lambda$ (solid), $6 \lambda$ (long dashed), and $12 \lambda$ (short dashed). Calculations are performed using Eq. (11) with a 256 point fast Hankel Transform routine (Ref. 10) with appropriate improvements (Ref. 11).

We recognize the integral above as a convolution of two terms. Letting

$$
\begin{aligned}
& F\left(-k_{x},-k_{y}\right) \\
& \quad=\Phi^{+}\left(-k_{x},-k_{y}\right) \exp \left[-j d\left(k_{x}^{2}+k_{y}^{2}\right) / 2 k_{0}\right],
\end{aligned}
$$

$G\left(k_{x}, k_{y}\right)=\Phi_{\mathrm{ro}}\left(k_{x}, k_{y}\right) \exp \left[j d\left(k_{x}^{2}+k_{y}^{2}\right) / 2 k_{0}\right]$,

we write

$$
\begin{aligned}
\Phi^{-}\left(k_{x}, k_{y}\right)= & \exp \left[-j d\left(k_{x}^{2}+k_{y}^{2}\right) / 2 k_{0}\right] \\
& \times\left[F\left(-k_{x},-k_{y}\right)^{*} G\left(k_{x}, k_{y}\right)\right],
\end{aligned}
$$

where ${ }^{*}$ denotes the convolution operation.

Combining this expression with Eq. (7) we find

$$
\begin{aligned}
V \cong & \frac{c_{11} k_{0}^{3}}{8 \pi^{2} \omega P} \int_{-\infty}^{\infty} \int F\left(-k_{x},-k_{y}\right) \\
& \times\left[F\left(-k_{x},-k_{y}\right)^{*} G\left(k_{x}, k_{y}\right)\right] d k_{x} d k_{y} .
\end{aligned}
$$

Let $f$ and $g$ show the inverse Fourier transform of $F$ and $G$, respectively. Application of the Parseval theorem on the last expression gives

$V=\frac{-2 \pi^{2} c_{11} k_{0}^{3}}{\omega P} \int_{-\infty}^{\infty} \int f(x, y) f(-x,-y) g(x, y) d x d y$,

where we use the relation $\mathscr{F}^{-1}\left[F\left(k_{x}, k_{y}\right) * G\left(k_{x}, k_{y}\right)\right]$
$=(2 \pi)^{2} f(x, y) g(x, y)$. Derivation leading to Eq. (10) utilizes paraxial approximation. Under this approximation the propagation in the angular spectrum domain is carried out by the exponential factor $\exp \left[-j d\left(k_{x}^{2}+k_{y}^{2}\right) / 2 k_{0}\right]$ instead of the nonparaxial $\exp \left(j k_{z} d\right)$. Using this fact and examining Eq. (9), one concludes that $f(x, y)$ is the acoustic potential generated by the transducer at $z=d$ plane in the absence of the spherical flaw. On the other hand $g(x, y)$ is an imaginary acoustic potential function at the $z=d$ plane that would generate the function $\phi_{\mathrm{ro}}(x, y)$, if it was propagated a distance $d$ toward the transducer. It is given by

$g(x, y)=\mathscr{F}^{-1}\left\{\Phi_{\text {го }}\left(k_{x}, k_{y}\right) \exp \left[j d\left(k_{x}^{2}+k_{y}^{2}\right) / 2 k_{0}\right]\right\}$.

Note that the formulation of the problem is such that the transducer is not necessarily centered on the $z$ axis.

Hence the problem of scanning the transducer with respect to flaw can be carried out easily if $f(x, y)$ is shifted properly. Let us redefine the function $f(x, y)$ as the acoustic potential at $z=d$ when the transducer is centered about the $z$ axis.

Now we can express the output voltage more appropriately in terms of the scan coordinates $\left(x_{0}, y_{0}\right)$ of the center of the transducer as follows:

$$
\begin{aligned}
V\left(x_{0}, y_{0}\right)= & -\frac{2 \pi^{2} c_{11} k_{0}^{3}}{\omega P} \int_{-\infty}^{\infty} \int f\left(x-x_{0}, y-y_{0}\right) \\
& \times f\left(-x-x_{0},-y-y_{0}\right) g(x, y) d x d y .
\end{aligned}
$$

If the output of the transducer is used to generate an image, $x_{0}, y_{0}$ are also the image coordinates. Equation (12) can, therefore, be used to predict the image.

We consider some limiting cases to understand the meaning of Eq. (12). If the spherical flaw is much smaller than the wavelength, a plane wave incident on the sphere will cause a spherically diverging scattered wave. Hence, $g(x, y)$ is a delta function $\delta(x, y)$ for a small scatterer. The output voltage is, therefore, proportional to $f^{2}\left(-x_{0},-y_{0}\right)$, the acoustic field intensity at the position of the flaw. This result is intuitively obvious. On the other hand, if the spherical flaw is not small compared to the wavelength, $g(x, y)$ has to be calculated numerically from Eq. (11). Figure 6 shows the result of the calculations for various sizes of a spherical cavity in a YAG crystal. The backscattered field $\phi_{\text {ro }}(x, y)$ is calculated with a computer program that uses the formulation of Johnson and Truel ${ }^{9}$ with appropriate corrections. Subsequently this potential distribution is propagated back to the central plane of the sphere. Due to the circular symmetry of the problem the propagation of the field is performed using a Fast Hankel transform routine. ${ }^{10,11}$

If the acoustic system is a focused system, such as the acoustic microscope ${ }^{12}$ with the focal plane adjusted to the central plane of the spherical flaw, then $f(x, y)$ will be confined to a narrow range. The function $g(x, y)$ can be wider than the function $f(x, y)$ for a large size spherical flaw. In this case the output of the acoustic microscope is written approximately as

$$
\begin{aligned}
V\left(x_{0}, y_{0}\right)= & K \int_{-\infty}^{\infty} \int f\left(x-x_{0}, y-y_{0}\right) \\
& \times f\left(-x-x_{0},-y-y_{0}\right) d x d y
\end{aligned}
$$


or

$$
V\left(x_{0}, y_{0}\right)=\left.K\left[f(x, y)^{*} f(x, y)\right]\right|_{x}=-2 x_{0} .
$$

We see that the image of a large spherical flaw as obtained from the acoustic microscope will be the convolution of the beam profile with itself. The maximum output voltage (brightest point in the acoustic image) will be obtained when the axis of the acoustic beam is aligned with the center of the sphere. This is obvious since the incident wavefronts match the curvature of the sphere surface when the alignment is satisfied. But the output voltage will be reduced quickly when the sphere is displaced slightly. The size of the bright spot in the image will be roughly equal to the size of the beam and is independent of the size of the sphere. For example, if an acoustic beam can be represented as a jinc function at its focal plane, the image of a large sphere obtained with that beam will be a jinc function that is half as narrow. This result follows from Eq. (13) and the fact that the convolution of a jinc function by itself gives the same jinc function.

\section{SUMMARY}

We have derived a backscattering formula that evaluates the output voltage of an acoustic transducer from an integral of the angular spectra of incident and reflected acoustic potentials on a plane. Since the quantities are in the angular spectrum domain, the propagation of waves are taken into account very easily. The expression reduces to a simple form when only the longitudinal waves are considered. Even though no examples involving shear waves are given, the application should be straightforward. Using the formulation, we have obtained a simple formula for the problem of a circular transducer facing a plane reflector. Use of the same formulation along with the paraxial approximation results in an expression that relates the output voltage due to a spherical scatterer as a function of the position of the scatterer with respect to the transducer. It is seen that for small scatterers the output voltage is proportional to the intensity of the acoustic field at the position of the scatterer. We have also found that for a scanned acoustic imaging system focused on the central plane of a large sphere, the output image is determined by the size of the focused acoustic beam rather than the size of the sphere.

\section{ACKNOWLEDGMENTS}

The author wishes to express his appreciation to $S$. Ayter and Prof. B. Auld for stimulating discussions that led to the derivation of the formula. He also wishes to thank Prof. C. F. Quate for suggestions and support; Ching-Hua Chov and Dr. B. Khuri-Yakub for discussions on the spherical cavity problem: Dr. V. Jipson for supplying the Fast Hankel Transform program; and S. Sheng for suggestions on the accuracy improvement of the same program. This work was supported by Air Force Office of Scientific Research under Contract F49620-78-C-0098.

\section{APPENDIX}

Generalized Parseval's Theorem ${ }^{13}$ : If $\mathscr{F}\left[f_{1}(x, y)\right]$ $=F_{1}\left(k_{x}, k_{y}\right)$ and $\mathscr{F}\left[f_{2}(x, y)\right]=F_{2}\left(k_{x}, k_{y}\right)$, then

$$
\begin{aligned}
& \int_{-\infty}^{\infty} \int f_{1}(x, y) f_{2}(x, y) d x d y \\
& \quad=\frac{1}{(2 \pi)^{2}} \int_{-\infty}^{\infty} \int F_{1}\left(k_{x}, k_{y}\right) F_{2}\left(-k_{x},-k_{y}\right) d k_{x} d k_{y}
\end{aligned}
$$

'G. S. Kino, J. Appl. Phys. 49, 3190 (1978).

${ }^{2}$ B. A. Auld, Wave Motion 1, 3 (1979).

${ }^{3}$ B. A. Auld, Acoustic Fields and Waves in Solids (Wiley, New York, 1973) ${ }^{4}$ L. M. Brekhovskikh, Waves in Layered Media (Academic, New York, 1960).

${ }^{5}$ J. W. Goodman, Introduction to Fourier Optics (McGraw-Hill, New York, 1968)

${ }^{6}$ M. McGuirk and C. K. Carniglia, J. Opt. Soc. Am. 67, 103 (1977).

${ }^{7}$ M. A. Breazeale, L. Adler, and G. W. Scott, J. Appl. Phys. 48, 530 (1977)

${ }^{8}$ A. Atalar, J. Appl. Phys. 49, 5130 (1978).

${ }^{9} \mathrm{G}$. Johnson and R. Truell, J. Appl. Phys. 36, 3466 (1965)

${ }^{10}$ A. E. Siegman, Opt. Lett. 1, 13 (1977).

${ }^{1} \mathrm{~S}-\mathrm{C}$. Cheng (private communication).

${ }^{12}$ C. F. Quate, A. Atalar, and H. K. Wickramasinghe, Proc. IEEE 67, 1092 (1979).

${ }^{13}$ R. N. Bracewell, The Fourier Transform and Its Applications (McGrawHill, New York, 1965). 